\title{
Alternate control delayed systems
}

\author{
Yuming Feng ${ }^{1,2}$, Dan $\mathrm{Tu}^{3}$, Chuandong $\mathrm{Li}^{1 *}$ and Tingwen Huang ${ }^{4}$
}

\author{
"Correspondence: licd@cqu.edu.cn \\ ${ }^{1}$ School of Electronic Information \\ Engineering, Southwest University, \\ Chongqing, 400715, P.R. China \\ Full list of author information is \\ available at the end of the article
}

\begin{abstract}
In the previous paper (Feng et al. in Adv. Differ. Equ. 2014:305, 2014), we have already used sandwich control to control a system. But when we considered the influence of delay, can sandwich control also be applied in the delayed system? In order to answer this question, we first introduce the alternate delayed system, then we study the exponential stability of delayed chaotic neural networks by means of alternate control. Some sufficient conditions are given in terms of a set of linear matrix inequalities to ensure the exponential stability of the system. Numerical simulations are presented to verify the correction of the obtained results.
\end{abstract}

Keywords: alternate control delayed system; globally exponential stabilization; Lu oscillator

\section{Introduction}

Alternate control [1] is a special case of switching control [2] and is a generalization of intermittent control [3, 4]. In an alternate control system, two different controls are applied alternately. So there is not rest time for the control. This system is suitable for the case in which the time is precious.

In [1] Feng et al. studied the alternate control system without delay. They have obtained some conditions in terms of LIMs to ensure the stability of the non-delayed system. For delayed systems [5-8], we know that the methods used are different from the ones without delay. There are many papers about delayed system [9-11]. A delayed system is much more difficult to study than the non-delayed one, we are trying to get some conditions to ensure the stability of the delayed system in the theory of control [12-14].

In this paper, we consider the influence of the delay of the system by means of alternate control, that is to say, we study the delayed system by means of alternate control. First of all, we introduce an alternate delayed system. Then we investigate the stability of it by constructing a Lyapunov function, and we obtain stability conditions in terms of LMIs. Lastly we study the stability of Lu oscillator by using the results obtained in the paper.

\section{Problem formulation and preliminaries}

Consider a class of delayed nonlinear systems described by

$$
\left\{\begin{array}{l}
\dot{x}(t)=A x(t)+f(x(t))+g(x(t-\tau))+u(t), \quad t>0, \\
x(t)=\phi(t), \quad t \in[-\tau, 0]
\end{array}\right.
$$

where $x \in R^{n}$ presents state vector, $f$ and $g$ are continuous nonlinear functions of $R^{n} \rightarrow R^{n}$ with $f(0)=g(0)=0$ and there exist two diagonal matrices $L_{1}=\operatorname{diag}\left(l_{1}^{(1)}, l_{2}^{(1)}, \ldots, l_{n}^{(1)}\right) \geq 0$

(C) 2015 Feng et al.; licensee Springer. This article is distributed under the terms of the Creative Commons Attribution 4.0 International License (http://creativecommons.org/licenses/by/4.0/), which permits unrestricted use, distribution, and reproduction in any medium, provided you give appropriate credit to the original author(s) and the source, provide a link to the Creative Commons license, and indicate if changes were made. 
and $L_{2}=\operatorname{diag}\left(l_{1}^{(2)}, l_{2}^{(2)}, \ldots, l_{n}^{(2)}\right) \geq 0$ such that $\|f(x)\|^{2} \leq x^{T} L_{1} x$ and $\|g(x)\|^{2} \leq x^{T} L_{2} x$ for any $x \in R^{n}, A \in R^{n \times n}$ is a constant matrix, $\phi$ is a function of $R^{n} \rightarrow R^{n}, u(t)$ denotes the external input of system (1).

For stabilizing the origin of system (1) by means of periodically alternate control, we assume that the control imposed on the system is of the following form:

$$
u(t)= \begin{cases}K_{1} x(t), & m T<t \leq m T+\theta \\ K_{2} x(t), & m T+\theta<t \leq(m+1) T\end{cases}
$$

where $K_{1}, K_{2} \in R^{n \times n}$ are constant matrices, $T>0$ denotes the control period, $\theta \in(0, T)$ is a constant.

Our target is to design suitable $T, \theta, K_{1}$ and $K_{2}$ such that system (1) can be stabilized at the origin.

By the control law (2), system (1) can be rewritten as follows with $m=0,1,2, \ldots$ :

$$
\begin{cases}\dot{x}(t)=A x(t)+f(x(t))+g(x(t-\tau))+K_{1} x(t), & m T<t \leq m T+\theta, \\ \dot{x}(t)=A x(t)+f(x(t))+g(x(t-\tau))+K_{2} x(t), & m T+\theta<t \leq(m+1) T, \\ x(t)=\phi(t), \quad t \in[-\tau, 0] . & \end{cases}
$$

It is obvious that system (3) is a classical switched system where the switching rule only depends on the time. Specifically, the switching rule of system (3) depends on $T$ and $\theta$. In the sequel, we will use the following definitions and lemmas.

Lemma 1 (Sanchez and Perez [15]) Given any real matrices $\Sigma_{1}, \Sigma_{2}, \Sigma_{3}$ of appropriate dimensions and a scalar $\epsilon \geq 0$ such that $0<\Sigma_{3}=\Sigma_{3}^{T}$, the following inequality holds:

$$
\Sigma_{1}^{T} \Sigma_{2}+\Sigma_{2}^{T} \Sigma_{1} \leq \epsilon \Sigma_{1}^{T} \Sigma_{3} \Sigma_{1}+\epsilon^{-1} \Sigma_{2}^{T} \Sigma_{3}^{-1} \Sigma_{2}
$$

Lemma 2 (Boyd et al. [16], Horn and Johnson [17]) The LMI

$$
\left[\begin{array}{cc}
Q(x) & S(x) \\
S^{T}(x) & R(x)
\end{array}\right]>0,
$$

where $Q(x)=Q^{T}(x), R(x)=R^{T}(x)$ and $S(x)$ depend affinely on $x$, is equivalent to

$$
R(x)>0, \quad Q(x)-S(x) R^{-1}(x) S^{T}(x)>0 .
$$

Definition 1 The zero solution of (1) is said to be globally exponentially stable if there are two constants $M(|\phi|)>0, \gamma>0$ such that

$$
\|x(t)\| \leq M(|\phi|) \exp (-\gamma t), \quad t>0
$$

where $|\phi|=\sup _{-\tau \leq t \leq 0}\|\phi(t)\|$.

Definition 2 Right-upper Dini's derivative of a function $V: R^{+} \times R^{n} \rightarrow R^{+}$is defined by

$$
D^{+} V(t, x(t))=\lim \sup \frac{1}{h}[V(t+h, x(t+h))-V(t, x(t)]
$$

Note that $V(x(t))$ or $V(x)$ is short for $V(t, x(t))$. 
Lemma 3 (Halany inequality [18]) Assume that $\tau>0$ and $\omega:[\mu-\tau, \infty) \rightarrow[0, \infty)$ is a continuous function such that

$$
\dot{\omega}(t) \leq-a \omega(t)+b \sup _{t-\tau \leq \theta \leq t} \omega(\theta)
$$

is satisfied for all $t \geq \mu$. If $a>b>0$, then

$$
\omega(t) \leq \bar{\omega}(\mu) \exp (-\gamma(t-\mu)), \quad t \geq \mu,
$$

where $\bar{\omega}(t)=\sup _{t-\tau \leq \theta \leq t} \omega(\theta)$ and $\gamma>0$ is the smallest real root of the equation

$$
a-b \exp (\gamma \tau)=\gamma
$$

Lemma 4 ([3]) Assume that $\tau>0$ and $\omega:[\mu-\tau, \infty) \rightarrow[0, \infty)$ is a continuous function such that

$$
\dot{\omega}(t) \leq a \omega(t)+b \omega(t-\tau)
$$

is satisfied for all $t \geq \mu$. If $a>0$ and $b>0$, then

$$
\omega(t) \leq \bar{\omega}(\mu) \exp (\eta(t-\mu+\tau)), \quad t \geq \mu,
$$

where $\bar{\omega}(t)=\sup _{t-\tau \leq \theta \leq t} \omega(\theta)$ and $\eta>0$ is the unique root of the equation

$$
a+b \exp (-\eta \tau)=\eta
$$

Throughout this paper, we use $P^{T}, \lambda_{M}(P)$ and $\lambda_{m}(P)$ to denote the transpose, the maximum eigenvalue and the minimum eigenvalue of a square matrix $P$, respectively. $\|x\|$ is used to denote the Euclidean norm of the vector $x$. The matrix norm $\|\cdot\|$ is also referred to as the Euclidean norm. We use $P>0(<0, \leq 0, \geq 0)$ to denote a symmetrical positive (negative, semi-negative, semi-positive) definite matrix $P . f\left(x\left(t_{1}^{-}\right)\right)$is defined by $f\left(x\left(t_{1}^{-}\right)\right)=\lim _{t \rightarrow t_{1}^{-}} f(x(t))$.

\section{Main results}

Theorem 1 If $\theta>\tau$ and there exist a symmetric and positive definite matrix $P \in R^{n \times n}$, positive scalar constants $g_{1}>0, g_{2}>0, q_{1}>0, q_{2}>0, \epsilon_{1}>0, \epsilon_{2}>0, \eta_{1}>0$ and $\eta_{2}>0$ such that the following hold:

(1) $P A+A^{T} P+P K_{1}+K_{1}^{T} P+\left(\epsilon_{1}+\eta_{1}\right) P^{2}+\epsilon_{1}^{-1} L_{1}+g_{1} P \leq 0$,

(2) $P A+A^{T} P+P K_{2}+K_{2}^{T} P+\left(\epsilon_{2}+\eta_{2}\right) P^{2}+\epsilon_{2}^{-1} L_{1}-g_{2} P \leq 0$,

(3) $\eta_{1}^{-1} L_{2}-q_{1} P \leq 0$,

(4) $\eta_{2}^{-1} L_{2}-q_{2} P \leq 0$

(5) $g_{1}>q_{1}$ and $\gamma(\theta-\tau)-\eta(T-\theta+\tau)>0$,

where $\gamma>0$ is the smallest real root of the equation $g_{1}-q_{1} \exp (\gamma \tau)=\gamma$ and $\eta>0$ is the unique root of the equation $g_{2}+q_{2} \exp (-\eta \tau)=\eta$, then the origin of system (3) is globally 
exponentially stable, and

$$
\|x(t)\|<\sqrt{\frac{\lambda_{M}(P)}{\lambda_{m}(P)}}|\phi| \exp \left(-(\gamma(\theta-\tau)-\eta(T-\theta+\tau)) \frac{t-\theta}{2 T}\right), \quad t>0,
$$

where $|\phi|=\sup _{-\tau \leq t \leq 0}\|\phi(t)\|$.

Proof Let us construct the following Lyapunov function:

$$
V(x(t))=x^{T}(x) P x(t)
$$

from which we obtain that

$$
\lambda_{m}(P)\|x(t)\|^{2} \leq V(x(t)) \leq \lambda_{M}(P)\|x(t)\|
$$

If $m T<t \leq m T+\theta$, then by (3), (4) and (5) we have that

$$
\begin{aligned}
\dot{V}(x(t))= & 2 x^{T}(t) P \dot{x}(t) \\
= & 2 x^{T}(t) P\left[A x(t)+f(x(t))+g(x(t-\tau))+K_{1} x(t)\right] \\
= & 2 x^{T}(t) P A x(t)+2 x^{T}(t) P f(x)+2 x^{T} P g(x(t-\tau))+2 x^{T} P K_{1} x(t) \\
= & x^{T}\left[P A+A^{T} P+P K_{1}+K_{1}^{T} P\right] x+2 x^{T} P f(x)+2 x^{T} P g(x(t-\tau)) \\
\leq & x^{T}\left[P A+A^{T} P+P K_{1}+K_{1}^{T} P\right] x \\
& +\epsilon_{1} x^{T}(t) P^{2} x(t)+\epsilon_{1}^{-1} x^{T}(t) L_{1} x(t) \\
& +\eta_{1} x^{T}(t) P^{2} x(t)+\eta_{1}^{-1} x^{T}(t-\tau) L_{2} x(t-\tau) \\
= & -g_{1} V(x(t))+x^{T}\left[P A+A^{T} P+P K_{1}+K_{1}^{T} P\right. \\
& \left.+\left(\epsilon_{1}+\eta_{1}\right) P^{2}+\epsilon_{1}^{-1} L_{1}+g_{1} P\right] x+q_{1} V(x(t-\tau)) \\
& +x^{T}(t-\tau)\left(\eta_{1}^{-1} L_{2}-q_{1} P\right) x(t-\tau) \\
\leq & -g_{1} V(x(t))+q_{1} V(x(t-\tau)),
\end{aligned}
$$

which implies that

$$
V(x(t)) \leq \bar{V}(x(m T)) \exp (-\gamma(t-m T))
$$

where $\gamma>0$ is the smallest real root of the equation $g_{1}-q_{1} \exp (\gamma \tau)=\gamma$.

Similarly, if $m T+\theta<t \leq(m+1) T$, then we have that

$$
\begin{aligned}
\dot{V}(x)= & 2 x^{T} P \dot{x} \\
\leq & g_{2} V(x(t))+x^{T}\left[P A+A^{T} P+P K_{2}+K_{2}^{T} P\right. \\
& \left.+\left(\epsilon_{2}+\eta_{2}\right) P^{2}+\epsilon_{2}^{-1} L_{1}-g_{2} P\right] x+q_{2} V(x(t-\tau)) \\
& +x^{T}(t-\tau)\left(\eta_{2}^{-1} L_{2}-q_{2} P\right) x(t-\tau) \\
\leq & g_{2} V(x(t))+q_{2} V(x(t-\tau)),
\end{aligned}
$$


which implies that

$$
V(x(t)) \leq \bar{V}(x(m T+\theta)) \exp (\eta(t-m T-\theta+\tau)),
$$

where $\eta>0$ is the unique root of the equation $g_{2}+q_{2} \exp (-\eta \tau)=\eta$.

It follows from (7) and (8) that

(1) If $0<t \leq \theta$, then we have that

$$
V(x(t)) \leq \bar{V}(x(0)) \exp (-\gamma t)
$$

So

$$
\bar{V}(x(\theta))=\sup _{\theta-\tau \leq t \leq \theta} V(t) \leq \sup _{\theta-\tau \leq t \leq \theta}(\bar{V}(x(0)) \exp (-\gamma t))=\bar{V}(x(0)) \exp (-\gamma(\theta-\tau)) .
$$

(2) If $\theta<t \leq T$, then we have that

$$
\begin{aligned}
V(x(t)) & \leq \bar{V}(x(\theta)) \exp (\eta(t-\theta+\tau)) \\
& \leq \bar{V}(x(0)) \exp (-\gamma(\theta-\tau)+\eta(t-\theta+\tau)) .
\end{aligned}
$$

So

$$
\bar{V}(x(T)) \leq \bar{V}(x(0)) \exp (-\gamma(\theta-\tau)+\eta(T-\theta+\tau)) .
$$

(3) If $T<t \leq T+\theta$, then we have that

$$
\begin{aligned}
V(x(t)) & \leq \bar{V}(x(T)) \exp (-\gamma(t-T)) \\
& \leq \bar{V}(x(0)) \exp (-\gamma(t-T)-\gamma(\theta-\tau)+\eta(T-\theta+\tau)) .
\end{aligned}
$$

So

$$
\bar{V}(x(T+\theta)) \leq \bar{V}(x(0)) \exp (-\gamma(2 \theta-2 \tau)+\eta(T-\theta+\tau)) .
$$

(4) If $T+\theta<t \leq 2 T$, then we have that

$$
\begin{aligned}
V(x(t)) & \leq \bar{V}(x(T+\theta)) \exp (\eta(t-T-\theta+\tau)) \\
& \leq \bar{V}(x(0)) \exp (\eta(t-T-\theta+\tau)-\gamma(2 \theta-2 \tau)+\eta(T-\theta+\tau)) .
\end{aligned}
$$

So

$$
\bar{V}(x(2 T)) \leq \bar{V}(x(0)) \exp (2 \eta(T-\theta+\tau)-2 \gamma(\theta-\tau)) .
$$

(5) If $2 T<t \leq 2 T+\theta$, then we have that

$$
\begin{aligned}
V(x(t)) & \leq \bar{V}(x(2 T)) \exp (-\gamma(t-2 T)) \\
& \leq \bar{V}(x(0)) \exp (-\gamma(t-2 T)+2 \eta(T-\theta+\tau)-\gamma(2 \theta-2 \tau)) .
\end{aligned}
$$


So

$\bar{V}(x(2 T+\theta) \leq \bar{V}(x(0)) \exp (2 \eta(T-\theta+\tau)-3 \gamma(\theta-\tau))$.

(6) If $2 T+\theta<t \leq 3 T$, then we have that

$$
\begin{aligned}
V(x(t)) & \leq \bar{V}(x(2 T+\theta)) \exp (\eta(t-2 T-\theta+\tau)) \\
& \leq \bar{V}(x(0)) \exp (\eta(t-2 T-\theta+\tau)+2 \eta(T-\theta+\tau)-3 \gamma(\theta-\tau)) .
\end{aligned}
$$

So

$\bar{V}(x(3 T)) \leq \bar{V}(x(0)) \exp (3 \eta(T-\theta+\tau)-3 \gamma(\theta-\tau))$.

By induction, we have that

(7) If $m T<t \leq m T+\theta$, i.e., $\frac{t-\theta}{T}<m \leq \frac{t}{T}$, then we have that

$$
V(x(t)) \leq \bar{V}(x(0)) \exp (-\gamma(t-m(T-\theta+\tau))+m \eta(T-\theta+\tau)) .
$$

(8) If $m T+\theta<t \leq(m+1) T$, i.e., $\frac{t}{T}<m+1 \leq \frac{t+T-\theta}{T}$, then we have that

$$
\begin{aligned}
V(x(t)) & \leq \bar{V}(x(0)) \exp (\eta(t-m T-\theta+\tau)+m \eta(T-\theta+\tau)-(m+1) \gamma(\theta-\tau)) \\
& =\bar{V}(x(0)) \exp (-\gamma(m+1)(\theta-\tau)+\eta(t-(m+1)(\theta-\tau))) .
\end{aligned}
$$

From (9) we know that

$$
\begin{aligned}
V(x(t)) & \leq \bar{V}(x(0)) \exp (-\gamma(t-m(T-\theta+\tau))+m \eta(T-\theta+\tau)) \\
& \leq \bar{V}(x(0)) \exp (-\gamma(m T-m(T-\theta+\tau))+m \eta(T-\theta+\tau)) \\
& =\bar{V}(x(0)) \exp (-(\gamma(\theta-\tau)-\eta(T-\theta+\tau)) m) \\
& <\bar{V}(x(0)) \exp \left(-(\gamma(\theta-\tau)-\eta(T-\theta+\tau)) \frac{t-\theta}{T}\right),
\end{aligned}
$$

where $m T<t \leq m T+\tau$.

From (10) we know that

$$
\begin{aligned}
V(x(t)) & \leq \bar{V}(x(0)) \exp (-\gamma(m+1)(\theta-\tau)+\eta(t-(m+1)(\theta-\tau))) \\
& \leq \bar{V}(x(0)) \exp (-\gamma(m+1)(\theta-\tau)+\eta((m+1) T-(m+1)(\theta-\tau))) \\
& =\bar{V}(x(0)) \exp (-(\gamma(\theta-\tau)-\eta(T-\theta+\tau))(m+1)) \\
& <\bar{V}(x(0)) \exp \left(-(\gamma(\theta-\tau)-\eta(T-\theta+\tau)) \frac{t}{T}\right) \\
& \leq \bar{V}(x(0)) \exp \left(-(\gamma(\theta-\tau)-\eta(T-\theta+\tau)) \frac{t-\theta}{T}\right)
\end{aligned}
$$

where $m T+\tau<t \leq(m+1) T$. 
It follows from (11) and (12) that, for any $t>0$,

$$
V(x(t))<\bar{V}(x(0)) \exp \left(-(\gamma(\theta-\tau)-\eta(T-\theta+\tau)) \frac{t-\theta}{T}\right) .
$$

By (5), (6) and (13), we conclude that

$$
\|x(t)\|<\sqrt{\frac{\lambda_{M}(P)}{\lambda_{m}(P)}}|\phi| \exp \left(-(\gamma(\theta-\tau)-\eta(T-\theta+\tau)) \frac{t-\theta}{2 T}\right), \quad t>0,
$$

where $|\phi|=\sup _{-\tau \leq t \leq 0}\|\phi(t)\|$.

So we finish the proof.

From Lemma 2, we know that the two conditions of Theorem 1 are equivalent to the following two LMIs, respectively:

$$
\begin{aligned}
& {\left[\begin{array}{cc}
P A+A^{T} P+P K_{1}+K_{1}^{T} P+\epsilon_{1}^{-1} L_{1}+g_{1} P & -P \\
-P & -\left(\epsilon_{1}+\eta_{1}\right)^{-1} I
\end{array}\right] \leq 0,} \\
& {\left[\begin{array}{cc}
P A+A^{T} P+P K_{2}+K_{2}^{T} P+\epsilon_{2}^{-1} L_{2}-g_{2} P & -P \\
-P & -\left(\epsilon_{2}+\eta_{2}\right)^{-1} I
\end{array}\right] \leq 0 .}
\end{aligned}
$$

Corollary 1 If $\theta>\tau$ and there exist a symmetric and positive definite matrix $P \in R^{n \times n}$, positive scalar constants $\epsilon_{1}>0, \epsilon_{2}>0, \eta_{1}>0, \eta_{2}>0, q_{1}>0, q_{2}>0$ and $\eta>0$ such that the following hold:

(1) $P A+A^{T} P+P K_{1}+K_{1}^{T} P+\left(\epsilon_{1}+\eta_{1}\right) P^{2}+\epsilon_{1}^{-1} L_{1}+g_{1} P \leq 0$, where $g_{1}=\gamma+q_{1} \exp (\gamma \tau)$ and $\gamma=\frac{\eta(T-\theta+\tau)}{\theta-\tau}+q_{1}$

(2) $P A+A^{T} P+P K_{2}+K_{2}^{T} P+\left(\epsilon_{2}+\eta_{2}\right) P^{2}+\epsilon_{2}^{-1} L_{1}-g_{2} P \leq 0$, where

$g_{2}=\eta-q_{2} \exp (-\eta \tau)>0$

(3) $\eta_{1}^{-1} L_{2}-q_{1} P \leq 0$,

(4) $\eta_{2}^{-1} L_{2}-q_{2} P \leq 0$, then the origin of system (3) is globally exponentially stable, and

$$
\|x(t)\|<\sqrt{\frac{\lambda_{M}(P)}{\lambda_{m}(P)}}|\phi| \exp \left(-(\gamma(\theta-\tau)-\eta(T-\theta+\tau)) \frac{t-\theta}{2 T}\right), \quad t>0,
$$

where $|\phi|=\sup _{-\tau \leq t \leq 0}\|\phi(t)\|$.

Proof In fact, the previous four conditions can imply

$$
g_{1}>q_{1}
$$

and

$$
\gamma(\theta-\tau)-\eta(T-\theta+\tau)>0
$$

From condition (1) we know

$$
g_{1}=\gamma+q_{1} \exp (\gamma \tau)=\frac{\eta(T-\theta+\tau)}{\theta-\tau}+q_{1}+q_{1} \exp (\gamma \tau)>q_{1}
$$


and

$$
\gamma=\frac{\eta(T-\theta+\tau)}{\theta-\tau}+q_{1}>\frac{\eta(T-\theta+\tau)}{\theta-\tau},
$$

which implies

$$
\gamma(\theta-\tau)-\eta(T-\theta+\tau)>0
$$

Thus, the fifth condition of Theorem 1 is valid. So the proof is completed.

Remark 1 In order to judge the global exponential stability of system (3), Corollary 1 needs to determine the existence of a symmetric and positive definite matrix $P \in R^{n \times n}$ and seven positive scalar constants $\epsilon_{1}, \epsilon_{2}, \eta_{1}, \eta_{2}, q_{1}, q_{2}$ and $\eta$ by the four linear matrix inequalities listed in it, while Theorem 1 has to determine the existence of a symmetric and positive definite matrix $P \in R^{n \times n}$ and eight positive scalar constants $\epsilon_{1}, \epsilon_{2}, \eta_{1}, \eta_{2}, q_{1}, q_{2}, g_{1}$ and $g_{2}$ by the five conditions of it. From this view of point, Corollary 1 is more applicative than Theorem 1.

\section{Numerical example}

Consider the neural oscillator model described by the following delayed differential equation:

$$
\dot{x}(t)=A x(t)+f(x(t))+g(x(t-1)),
$$

where

$$
A=\left(\begin{array}{cc}
-1 & 0 \\
0 & -1
\end{array}\right), \quad f(x(t))=\left(\begin{array}{cc}
2 & -0.1 \\
-5 & 3
\end{array}\right)\left(\begin{array}{l}
\tanh x_{1}(t) \\
\tanh x_{2}(t)
\end{array}\right)
$$

and

$$
g(x(t-1))=\left(\begin{array}{ll}
-1.5 & -0.1 \\
-0.2 & -2.5
\end{array}\right)\left(\begin{array}{l}
\tanh x_{1}(t-1) \\
\tanh x_{2}(t-1)
\end{array}\right) .
$$

This model was named Lu oscillator [19] and it is shown to be chaotic as in Figure 1. The time response curves are shown in Figure 2.

It is easy to obtain that

$$
\begin{aligned}
\|f(x(t))\|^{2} & =f^{T}(x(t)) f(x(t)) \\
& =\left(\begin{array}{l}
\tanh x_{1}(t) \\
\tanh x_{2}(t)
\end{array}\right)^{T}\left(\begin{array}{cc}
2 & -0.1 \\
-5 & 3
\end{array}\right)^{T}\left(\begin{array}{cc}
2 & -0.1 \\
-5 & 3
\end{array}\right)\left(\begin{array}{l}
\tanh x_{1}(t) \\
\tanh x_{2}(t)
\end{array}\right) \\
& \leq x^{T} L_{1} x,
\end{aligned}
$$

where $L_{1}=\left(\begin{array}{cc}29 & -15.2 \\ -15.2 & 9.01\end{array}\right)$.

Similarly, we can get that $\|g(x(t))\|^{2} \leq x^{T} L_{2} x$, where $L_{2}=\left(\begin{array}{ll}2.29 & 0.65 \\ 0.65 & 6.26\end{array}\right)$.

Next, we will use Theorem 1 to judge the global exponential stability of system (16). 


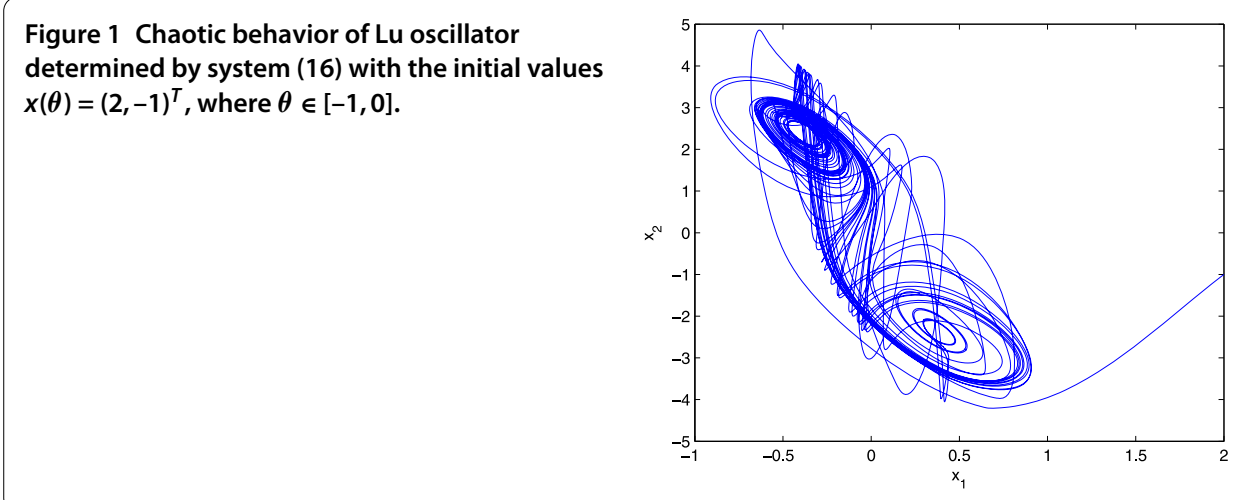

Figure 2 Time response curves of Lu oscillator without control when the initial values are $x(\theta)$ $=(2,-1)^{T}$, where $\theta \in[-1,0]$.

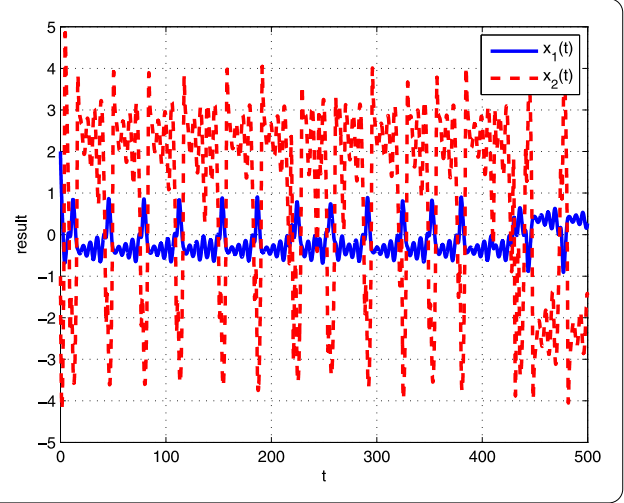

\section{Choosing}

$$
\begin{aligned}
& K_{1}=\operatorname{diag}(-25,-25), \\
& K_{2}=\operatorname{diag}(-10,-10),
\end{aligned}
$$

with $T=3$ and $\theta=1.5$, solving LMIs (14), (15), $\eta_{1}^{-1} L_{2}-q_{1} P \leq 0, \eta_{2}^{-1} L_{2}-q_{2} P \leq 0$ and inequalities $g_{1}>q_{1}, \gamma(\theta-1)-\eta(T-\theta+1)>0$, where $\gamma>0$ is the smallest real root of the equation $g_{1}-q_{1} \exp (\gamma)=\gamma$ and $\eta>0$ is the unique root of the equation $g_{2}+q_{2} \exp (-\eta)=\eta$, we obtain a feasible solution:

$$
\begin{aligned}
& \epsilon_{1}=8.6, \quad \epsilon_{2}=0.6, \quad \eta_{1}=9, \quad \eta_{2}=11, \quad g_{1}=28.2703, \quad g_{2}=0.3211, \\
& q_{1}=0.72, \quad q_{2}=0.63,
\end{aligned}
$$

and

$$
P=\left[\begin{array}{ll}
0.9498 & 0.0049 \\
0.0049 & 1.0066
\end{array}\right]
$$

Thus by Theorem 1 we obtain that the origin of system (3) is globally exponentially stable. The time response curves of Lu oscillator with alternate control are shown in Figure 3, while Figure 4 shows the corresponding control signal.

In the following, we will apply Corollary 1 to determine the global exponential stability of system (16). 


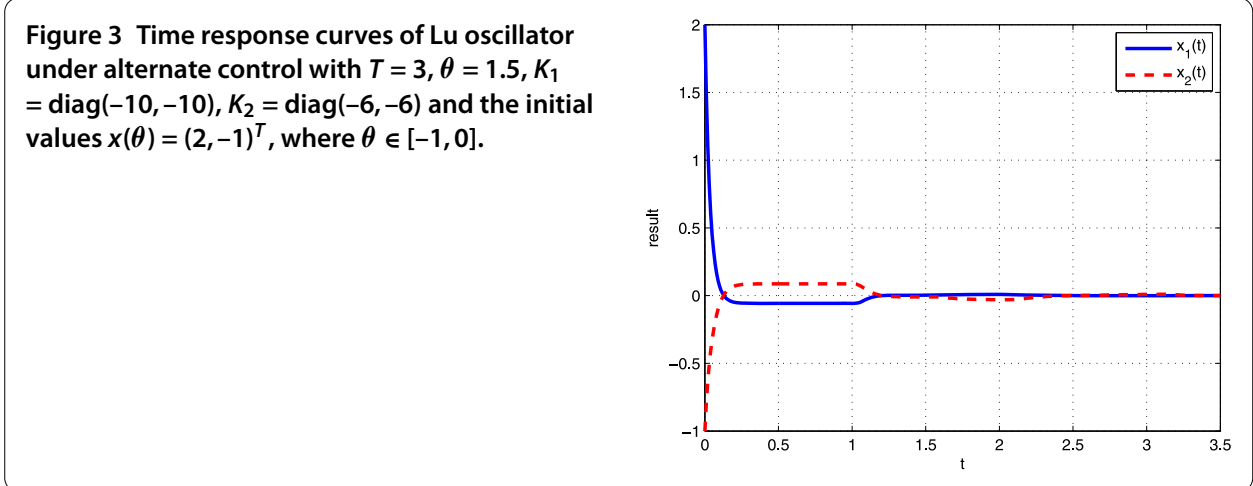

Figure 4 Control signal for Lu oscillator of $T=3$, $\theta=1.5, K_{1}=\operatorname{diag}(-10,-10)$ and $K_{2}=\operatorname{diag}(-6,-6)$.

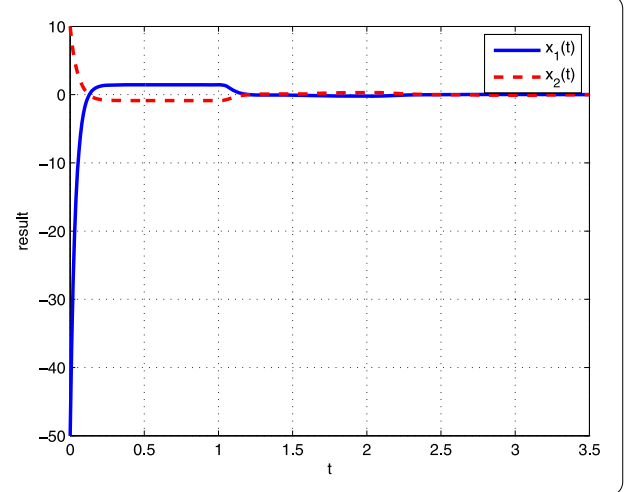

Choosing

$$
\begin{aligned}
& K_{1}=\operatorname{diag}(-15,-15), \\
& K_{2}=\operatorname{diag}(-9,-9),
\end{aligned}
$$

with $T=3$ and $\theta=1.5$, solving LMIs (14), (15), $\eta_{1}^{-1} L_{2}-q_{1} P \leq 0$ and $\eta_{2}^{-1} L_{2}-q_{2} P \leq 0$, where $g_{1}=\gamma+q_{1} \exp (\gamma \tau)$ and $\gamma=\frac{\eta(T-\theta+\tau)}{\theta-\tau}+q_{1}$ and $g_{2}=\eta-q_{2} \exp (-\eta \tau)>0$, we obtain a feasible solution:

$$
\begin{aligned}
& \epsilon_{1}=4.6, \quad \epsilon_{2}=0.6, \quad \eta_{1}=9, \quad \eta_{2}=11, \\
& q_{1}=0.52, \quad q_{2}=0.43, \quad \eta=0.45
\end{aligned}
$$

and

$$
P=\left[\begin{array}{ll}
1.2212 & 0.0532 \\
0.0532 & 1.3590
\end{array}\right] .
$$

Thus by Corollary 1 we obtain that the origin of system (16) is globally exponentially stable. The time response curves of Lu oscillator with alternate control are shown in Figure 5. The control signal is shown in Figure 6. 
Figure 5 Time response curves of Lu oscillator $=\operatorname{diag}(-15,-15), K_{2}=\operatorname{diag}(-11,-11)$ and the initial values $x(\theta)=(2,-1)^{T}$, where $\theta \in[-1,0]$. under alternate control with $T=3, \theta=1.5, K_{1}$

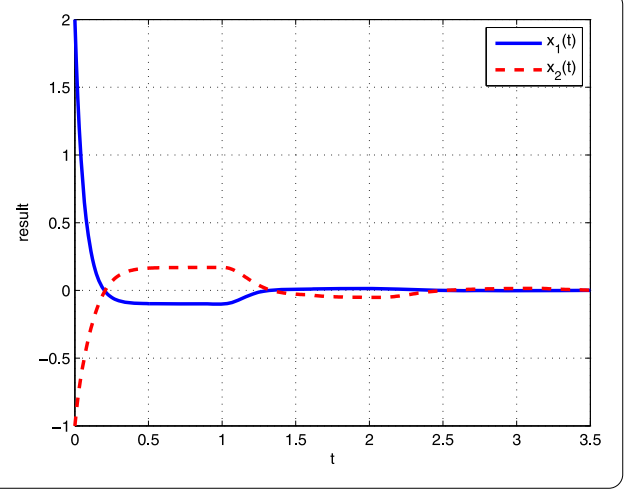

Figure 6 Control signal for Lu oscillator of $T=3$, $\theta=1.5, K_{1}=\operatorname{diag}(-15,-15)$ and $K_{2}=\operatorname{diag}(-11$, $-11)$.

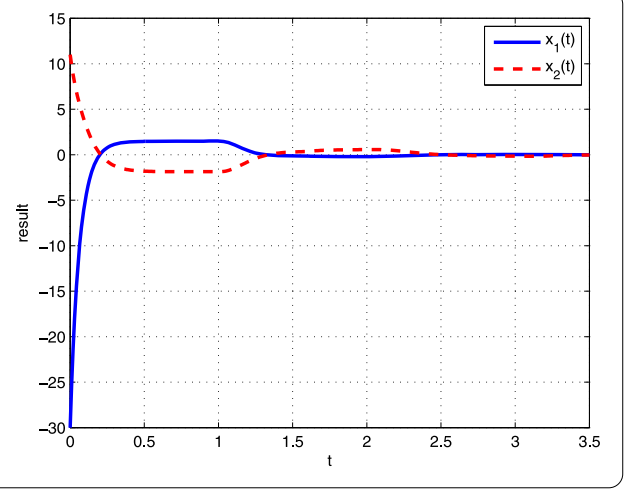

\section{Conclusions}

This paper studies the delayed system by using alternate control method. Some conditions to ensure the stability of the system are given in terms of linear matrix inequalities. By the results obtained, the Lu oscillate is controlled.

\section{Competing interests}

The authors declare that they have no competing interests.

\section{Authors' contributions}

The ideal of alternate control delayed system was proposed by $\mathrm{CL}$ and $\mathrm{YF}$. The main theory was proved by $\mathrm{YF}$ and DT. The paper was typed by $\mathrm{YF}$ and $\mathrm{TH}$ and all the figures were provided by TH. All authors read and approved the final manuscript.

\section{Author details}

${ }^{1}$ School of Electronic Information Engineering, Southwest University, Chongqing, 400715, P.R. China. ${ }^{2}$ School of Mathematics and Statistics, Chongqing Three Gorges University, Wanzhou, Chongqing, 404100, P.R. China. ${ }^{3}$ Department of Mathematics, Texas A\&M University at Qatar, P.O. Box 23874, Doha, Qatar. ${ }^{4}$ School of Physical Education, Southwest University, Chongqing, 400715, P.R. China.

\section{Acknowledgements}

This research is supported by the Natural Science Foundation of China (Grant No: 61374078), NPRP Grant No. NPRP 4-1162-1-181 from the Qatar National Research Fund (a member of Qatar Foundation), Scientific \& Technological Research Foundation of Chongqing Municipal Education Commission (Grant Nos. KJ1401006, KJ1401019) and the Fundamental Research Funds for the Central Universities (Grant No. XDJK2015D004).

Received: 2 December 2014 Accepted: 24 April 2015 Published online: 07 May 2015

\section{References}

1. Feng, Y, et al.: Alternate control systems. Adv. Differ. Equ. 2014, 305 (2014)

2. Tanwani, A, Shim, H, Liberzon, D: Observability for switched linear systems: characterization and observer design. IEEE Trans. Autom. Control 58(4), 891-904 (2013)

3. Huang, J, Li, C, Han, Q: Stabilization of delayed chaotic neural networks by periodically intermittent control. Circuits Syst. Signal Process. 28, 567-579 (2009) 
4. Huang, T, Li, C, Liu, X: Synchronization of chaotic systems with delay using intermittent linear state feedback. Chaos $18,033122(2008)$

5. Xia, W, Cao, J: Pinning synchronization of delayed dynamical networks via periodically intermittent control. Chaos 19 , 013120 (2009). doi:10.1063/1.3071933

6. Yang, X, Cao, J: Stochastic synchronization of coupled neural networks with intermittent control. Phys. Lett. A 373(36), 3259-3272 (2009)

7. Yang, X: Can neural networks with arbitrary delays be finite-timely synchronized? Neurocomputing 143, 275-281 (2014)

8. Zheng, G, Cao, J: Robust synchronization of coupled neural networks with mixed delays and uncertain parameters by intermittent pinning control. Neurocomputing 141(2), 153-159 (2014)

9. El'sgol'ts, LE, Norkin, SB: Introduction to the Theory and Application of Differential Equations with Deviating Arguments. Academic Press, New York (1973)

10. Liao, X, Wang, L, Yu, P: Stability of Dynamical Systems. Monograph Series on Nonlinear Science and Complexity. Elsevier, Amsterdam (2007)

11. Liao, X, Yu, P: Absolute Stability of Nonlinear Control Systems. Springer, New York (2008)

12. Yu, J, Liu, L, Wang, L, Tan, M, Xu, D: Turning control of a multilink biomimetic robotic fish. IEEE Trans. Robot. 24(1), 201-206 (2008)

13. Shen, $Y, X u, D, T a n, M, Y u$, J: Mixed visual control method for robots with self-calibrated stereo rig. IEEE Trans. Instrum. Meas. 59(2), 470-479 (2010)

14. Dong, D, Petersen, IR: Quantum control theory and applications: a survey. IET Control Theory Appl. 4(12), 2651-2671 (2010)

15. Sanchez, EN, Perez, JP: Input-to-state stability (ISS) analysis for dynamic NN. IEEE Trans. Circuits Syst. I, Regul. Pap. 46(11), 1395-1398 (1999)

16. Boyd, S, Ghaoui, L, Feron, EEl, Balakrishnan, V: Linear matrix inequalities in system and control theory. In: Linear Matrix Inequalities in System and Control Theory. SIAM, Philadephia (1994)

17. Horn, R, Johnson, C: Matrix Analysis. Cambridge University Press, Cambridge (1985)

18. Halanay, A: Differential Equations: Stability, Oscillations, Time Lags. Academic Press, New York (1966)

19. Lu, H: Chaotic attractors in delayed neural networks. Phys. Lett. A 298, 109-116 (2002)

\section{Submit your manuscript to a SpringerOpen ${ }^{\circ}$ journal and benefit from:}

- Convenient online submission

Rigorous peer review

- Immediate publication on acceptance

- Open access: articles freely available online

- High visibility within the field

- Retaining the copyright to your article 